\title{
考虑溶质原子拖拽效应及各向异性的元胞自动机法模拟 钛合金单相区静态粗化
}

\author{
武川，杨合*，李宏伟，㚞晓光 \\ 西北工业大学材料科学与工程学院, 凝固技术国家重点实验室, 西安 710072 \\ * 联系人, E-mail: yanghe@nwpu.edu.cn
}

2011-09-06 收稿, 2011-11-22 接受

国家自然科学基金重点项目(50935007)、国家重点基础研究发展计划(2010CB731701)、西北工业大学凝固技术国家重点实验室研究基金 (27-TZ-2010)和先进材料及其成形技术学科创新引智基地(B08040)资助

\begin{abstract}
摘要 晶粒静态粗化是一种重要的物理现象, 严重影响微观结构的演化和材料的力学性 能. 如何有效模拟这一过程已经成为该领域的研究热点. 本文通过考虑溶质原子拖拽效 应和晶界迁移各向异性对晶粒粗化的影响, 建立了元胞自动机模型, 并对钛合金单相区 静态粗化过程进行了模拟. 为了描述不同溶质原子的拖拽效应对粗化的影响, 文中将溶 质原子在 beta相中的扩散速度等效转化成钛原子的迁移速度. 为此, 提出了定量描述溶质 原子扩散速度与钛原子迁移速度之间转化关系的数学表达式. 其中, 表达式中考虑了影 响溶质原子扩散速度的因素如溶质原子的半径、原子质量及晶格类型. 通过引入参数 $c_{0}$ 考虑晶界迁移各向异性对粗化的影响. 当 $c_{0}$ 为 1 时, 则认为晶界迁移为各向异性, 若为 0 时则认为是各向同性. 将上述表达式应用到元胞自动机模型中，模拟钛合金(包括 TC4, Ti17, TG6 和 TA15)在单相区的静态粗化现象. 预测结果包括粗化动力学和组织演化与试 验进行了对比, 而且较为吻合. 最后, 讨论了时间、温度和化学成分对粗化的影响, 以及 本文元胞自动机模型预测粗化的局限性.
\end{abstract}

\section{关键词}

晶粒粗化

元胞自动机模型

溶质原子拖拽效应

晶界迁移各向异性

钛合金
钛合金材料的力学性能如抗蠕变性能、断裂韧 性及裂纹难扩展性直接受到单相区 (beta 区) 晶粒尺寸 的影响 ${ }^{[1,2]}$. 然而在高温下, 受 beta 钛的结构特点和 相对高的层错能的影响, beta 区晶粒极易发生静态粗 化现象 ${ }^{[3]}$, 这将极大降低构件的力学性能. 因此, 为 了获得构件的预期力学性能, 有必要理解钛合金单 相区晶粒静态粗化过程, 以控制粗化现象对材料力 学性能的影响.

在过去的十几年, 许多学者已经通过大量的试 验研究了钛合金在单相区的静态粗化现象. Ivasishin 等人 ${ }^{[4,5]}$ 系统地研究了织构演化对 TC4 钛合金单相区 晶粒粗化指数 $n$ 及激活能的影响. 研究结果表明, 粗化指数 $n$ 值范围在 $0.22 ~ 0.31$ 之间. 但是对于不同
的材料或试验条件, $n$ 值的差别较大. 近年来, Semiatin 等人 ${ }^{[6-8]}$ 通过大量热处理试验研究了 TC4 钛合金 的静态粗化现象. 他们发现, 初生 alpha 相晶粒的粗 化由溶质原子的扩散所控制, 而且粗化指数 $n$ 为 3 . 同样, Bradley 等人 ${ }^{[9]}$ 及 Cotrina 等人 ${ }^{[10]}$ 通过试验手段 研究了晶粒的粗化现象.

近年来, 各种类型的数值模拟手段如波茨-蒙特 卡洛 $(\mathrm{MC})$ 法 $^{[11 \sim 13]}$ 、相场法 ${ }^{[14,15]}$ 及元胞自动机法 ${ }^{[16 ~ 18]}$ 已经被逐渐应用到微观组织演化的模拟中 ${ }^{[19]}$. 元胞 自动机模拟法由于自身的优点, 得到了更加广泛的 应用 ${ }^{[20]}$. Geiger 等人 ${ }^{[21]}$ 建立了元胞自动机模型, 模拟 了晶粒的粗化现象, 并且讨论了晶粒取向差、晶界 原子扩散激活能及界面能对粗化的影响. 随后,

英文版见: Wu C, Yang H, Li H W, et al. Static coarsening of titanium alloys in single field by cellular automaton model considering solute drag and anisotropic mobility of grain boundaries. Chin Sci Bull, 2012, 57, doi: 10.1007/s11434-012-5002-9 
Raghavan 等人 ${ }^{[22,23]}$ 和 Kugler 等人 ${ }^{[24]}$ 模拟了多晶材 料晶粒粗化过程中动力学和拓扑学特征. 然而, 大多 数元胞自动机模型忽略了溶质原子的拖拽效应及晶 界迁移各向异性对粗化的影响, 以至于模拟结果包 括粗化指数和拓扑结构的演化与试验值存在较大 误差.

溶质原子的拖拽效应和晶界迁移各向异性影响 晶粒粗化过程. 溶质原子在基体中的扩散速度受到 半径、质量及晶格类型的影响. 如果溶质原子的扩散 速度远远小于基体原子的迁移速度, 则晶界处溶质 原子将阻碍基体晶界的迁移，拖拽效应对基体晶粒 的粗化影响较为明显. 由于晶粒取向差的影响, 对于 不同晶粒，其晶界迁移率不同，这将影响晶粒的粗化 过程. 然而，在过去的十几年中，模拟晶粒粗化的元 胞自动机模型忽略了溶质原子拖拽效应及各向异性 的影响, 因此模拟结果与试验值存在较大误差. 因此, 本研究通过考虑不同类型溶质原子的拖拽效 应及晶界迁移率各向异性的影响, 建立元胞自动机 模型模拟钛合金单相区的静态粗化现象. 本文首先 提出了定量描述溶质原子扩散速度与钛原子迁移速 度之间关系的数学表达式, 其次通过参数 $c_{0}$ 的引人 来考虑晶界迁移各向异性对粗化的影响, 最后将上 述表达式应用到元胞自动机模型中模拟粗化过程.

\section{1 元胞自动机模型}

\section{1 晶粒静态粗化模型}

晶粒静态粗化是一个热激活过程，对于铁碳合 金的粗化现象已经有了大量研究 ${ }^{[25]}$. 通常情况下, 晶粒粗化由晶界处原子的跃迁和晶界迁移两个过程 完成. 只有当晶界处原子具有足够高的能量才可能 克服势垒的约束从晶界的一侧跃迁到另一侧 ${ }^{[26]}$. 类 似地, 对于元胞自动机模拟晶粒粗化过程, 位于晶界 处的元胞以概率 $p$ 发生跃迁. 根据麦克斯韦-波尔兹曼 分布定律, 晶界处元胞跃迁概率 $p$ 由方程(1)得到,

$$
\begin{gathered}
p=\frac{\Delta Q}{\Delta Q_{\max }}, \Delta Q>0, \\
p=\frac{\exp \left(-\frac{Q_{b}}{R T}\right)}{\exp \left(-\frac{Q_{b}}{R T}\right)+\exp \left(-\frac{E}{R T}\right)}, \Delta Q \leqslant 0,
\end{gathered}
$$

式中, $\Delta Q$ 为元胞 $i$ 总能量与自扩散激活能 $Q_{b}$ 之差. 元
胞总能量包括系统热能 $E^{t}$ 与总的边界能 $E^{b}$. 其中元 胞 $i$ 的热能由方程(2)求得 ${ }^{[27]}$,

$$
E_{i}^{t}=-R T \ln r,
$$

式中, $r(0<r<1)$ 为一随机数, 由计算机程序生成, $R$ 和 $T$ 分别为气体体积常数和系统温度. 相邻两元胞 $i, j$ 界面能 $\gamma_{i j}$ 由 Read-Shockley 方程计算得到 ${ }^{[28]}$,

$$
\gamma_{i j}=\left\{\begin{array}{cc}
\gamma_{m}, & \theta_{i j} \geqslant \theta_{m} \\
\gamma_{i j}=\gamma_{m} \frac{\theta_{i j}}{\theta_{m}}\left[1-\ln \left(\frac{\theta_{i j}}{\theta_{m}}\right)\right], & \theta_{i j}<\theta_{m},
\end{array}\right.
$$

式中, $\theta_{m}$ 为高角度晶界取向差, 通常设为 $15^{\circ}, \theta_{i j}$ 为相 邻元胞取向差, $\gamma_{m}$ 为高角度晶界边界能, 由方程(4)计 算可得 ${ }^{[29]}$

$$
\gamma_{m}=\frac{\mu b \theta_{m}}{4 \pi(1-v)},
$$

式中, $\mu$ 为材料剪切模量, $b$ 为伯格斯矢量, $v$ 为泊松比. 元胞 $i$ 总的界面能为

$$
E_{i}^{b}=\sum_{j}^{N} \gamma_{i j},
$$

式中, $N$ 为与元胞 $i$ 相邻元胞总数, 由所选邻居类型决 定. 元胞 $i$ 的邻居元胞总数为 4 .

晶界迁移是一个基于曲率驱动的过程. 其中, 驱 动力是由两相邻晶粒的能量差提供. 因此, 可以认为 在粗化过程中, 晶界的迁移速度 $v$ 可表示为

$$
v=m f,
$$

式中, $m, f$ 分别为晶界迁移率和晶粒粗化驱动力. 其 中驱动力 $f_{i j}$ 可表示为

$$
f_{i j}=\Delta E / R_{i j},
$$

式中, $\Delta E$ 为两相邻元胞 $i, j$ 的总的能量差值, $R_{i j}$ 为晶 粒半径. 在 $\Delta t$ 时间内, 晶界处元胞 $i$ 沿着元胞 $j$ 方向 长大距离 $l$ 可以表示为

$$
l_{i j}(t+\Delta t)=l_{i j}(t)+v_{i j} \Delta t,
$$

式中, $l_{i j}(t)$ 为元胞 $i$ 在时间 $t$ 的长大距离, 其初始值为 0. $l_{i j}(t+\Delta t)$ 为元胞 $t+\Delta t$ 时刻长大距离. Kugler 等人 ${ }^{[30]}$ 定义了时间增量 $\Delta t$ 与元胞尺寸 $d$ 及晶界元胞最大迁 移速率的关系. 根据式(6) (8), 当晶界能 $\gamma_{i j}$ 和晶粒半 径 $R_{i j}$ 分别取最大值 $\gamma_{m}$ 和初始值 $R_{0}$ 时, 晶界迁移速率 为最大. 因此, 时间增量 $\Delta t$ 可表示为

$$
\Delta t=C(T) \frac{d}{v_{\text {max }}}=C(T) \frac{d \cdot R_{0}}{m\left(\gamma_{m}+E_{i j}^{t}\right)},
$$

式中, $d$ 为元胞尺寸, $R_{0}$ 为初始晶粒尺寸, $C(T)$ 为与温 度相关的材料参数. 


\section{2 溶质原子拖拽效应的定量描述}

由于晶界结构缺陷的存在, 晶界附近溶质原子 的浓度要高于晶粒内部的溶质浓度. 由于浓度梯度 的存在, 溶质原子的扩散成为可能, 同时, 钛原子 (基体)发生扩散或者晶界迁移. 如果溶质原子的扩散 速度小于钛原子的迁移速度(晶界迁移速度), 溶质原 子将阻碍晶界的运动. 钛中的合金元素主要包括 alpha-钛的稳定元素, 如铝元素(Al), beta-钛的稳定元 素, 如钒元素 $(V)$ 、钼元素 $(M o)$ 和锡元素 $(S n)$. 这些 元素在 beta-钛中的扩散速度受溶质原子自身的半 径、质量及晶格类型的影响. 为了定量描述溶质原子 的拖拽效应，应将不同溶质原子的扩散速度等效地 转化成钛原子的迁移速度. 为此, 本文提出了一个描 述它们之间转化关系的数学表达式. 假定认为溶质 原子的扩散速度与钛原子的扩散速度之比与溶质原 子半径、质量与钛原子的半径、质量成反比. 那么, 可 以表示为

$$
\frac{v_{i}}{v_{\mathrm{Ti}}}=\frac{h}{\chi_{1} \cdot \chi_{2}},
$$

式中, $v_{i}$ 与 $v_{\mathrm{Ti}}$ 分别为溶质原子和钛原子的扩散速度. $h$ 参数反映溶质原子晶格类型对粗化的影响. 如果溶 质原子晶格类型与 beta-钛原子的类型相同, 如 $\mathrm{V}$, $\mathrm{Mo}, \mathrm{Sn}$ 元素均为体心立方晶格, 则 $h$ 取值为 1 , 否则, $h$ 设置为小于 $1 . \chi_{1}$ 与 $\chi_{2}$ 分别为溶质原子的半径、质 量与钛原子的比值, 分别表示为

$$
\begin{aligned}
& \chi_{1}=\frac{R_{i}}{R_{\mathrm{Ti}}}, \\
& \chi_{2}=\frac{m_{i}}{m_{\mathrm{Ti}}},
\end{aligned}
$$

式中, $R_{i}$ 为溶质原子半径, $R_{\mathrm{Ti}}$ 为钛原子半径, $m_{i}$ 为 溶质原子质量, $m_{\mathrm{Ti}}$ 为钛原子质量. 如果 $v_{i} / v_{\mathrm{Ti}}$ 大于 1 , 那么溶质原子的扩散速度大于钛原子的扩散速度, 此时溶质原子的拖拽效应可以忽略不计. 相反, 若 $v_{i} / v_{\mathrm{Ti}}$ 小于 1 , 则溶质原子的扩散速度小于钛原子的 迁移速度，此时溶质原子阻碍晶界的迁移，拖拽效应 不可忽略. 为了在元胞自动机模型中考虑溶质原子 拖拽效应对粗化的影响, 将制定相应的元胞转化规 则, 其详细步骤在 1.4 节中阐述.

\section{3 晶界迁移率各相异性}

在传统元胞自动机模型中, 一般假设晶界迁移
率与晶粒间取向差无关，即各向同性. 在这种条件下, 元胞自动机模拟粗化过程中拓扑特征的演化与试验 观察不同．模拟所得晶粒尺寸的分布特征呈现 “左 倾” 趋势, 而不是试验所得的正态分布 ${ }^{[31]}$. 本文认为 晶界迁移率与取向差相关，呈现各向异性. 因此，我 们引人参数 $c_{0}$ 来考虑晶粒取向差对迁移率的影响. 相邻元胞 $i, j$ 晶界迁移率 $m_{i j}$ 可以表示为 ${ }^{[32]}$

$$
m_{i j}=m_{0}\left\{1-c_{0} \cdot \exp \left[-\left(\frac{\theta_{i j}}{10}\right)^{3}\right]\right\},
$$

式中, 高角度晶界迁移率 $m_{0}$ 为常数. 参数 $c_{0}$ 取值为 1 , 为晶界迁移率各向异性, $c_{0}$ 取值为 0 时, 为晶界迁 移率各向同性.

\section{4 元胞自动机模拟晶粒静态粗化步骤}

本文通过饱和形核及等轴长大的元胞自动机模 型获得等轴状初始组织. 元胞状态变量包括晶粒取 向状态变量, 能量状态变量 (系统总能量、热能及晶 界能), 扩散速度变量, 位置变量和属性变量. 元胞 尺寸的选取由初始晶粒尺寸决定.

元胞取向状态变量的取值范围为 $0^{\circ} \sim 180^{\circ}$, 通过 计算机随机产生. 在每一模拟步骤中, 计算出晶界元 胞的能量及速度变量, 并赋给每一元胞. 位置变量用 于指定元胞位于晶粒内部(位置变量取值为 1 )或者晶 界处(位置变量取值为 0 ). 属性变量用于指定元胞是 否属于钛原子(属性值为 0 )或者其他溶质原子(属性 值大于 0 ).

在每一个模拟步骤, 根据元胞转化规则, 元胞状 态变量发生改变. 同时, 元胞模拟微观组织演化. 元 胞自动机模拟晶粒粗化的完整过程主要包括晶界处 元胞的跃迁及晶界的迁移，具体过程如图 1 所示.

（1）跃迁. 通过程序，首先选取位于晶界处的 元胞. 根据方程(2) (4), 计算晶界元胞的热能和晶界 能, 以此计算元胞的总能量(方程(5)). 将晶界处元胞 总能量与扩散激活能做差比较, 若差值大于 0 , 则元 胞跃迁的概率按照方程 1 (a)计算, 若差值小于 0 , 则 按照方程 1(b)计算. 同时, 计算机程序生成一随机数 $r(0<r<1)$, 若通过方程 $(1)$ 计算所得的概率 $p$ 不小于 $r$ 值，则元胞可以发生跃迁.

（2）晶界迁移. 对于发生跃迁的元胞，程序首 先判断跃迁元胞的邻居元胞的属性变量值. 如果邻 居元胞被代表溶质原子集合的溶质元胞占有(属性 


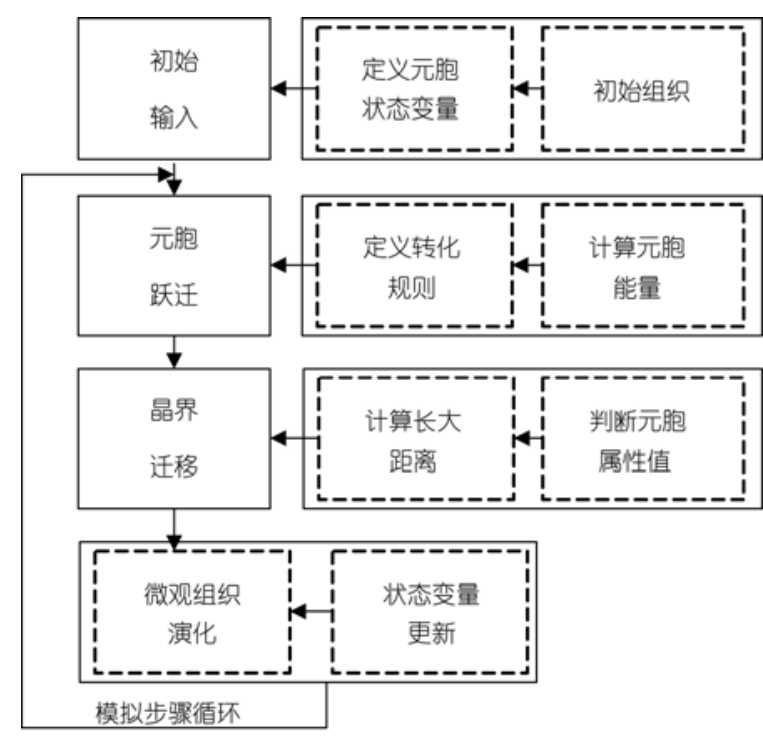

图 1 元胞自动机模拟晶粒粗化过程

变量值大于 0 ), 则晶界迁移过程可能受到溶质原子 拖拽的影响. 为了在元胞自动机模型中考虑这种效 应, 粗化过程中的元胞转化规则如下: 首先根据方程 (10)将特定溶质原子(元胞)的扩散速度等效地转化成 钛原子的扩散速度, 然后与钛原子的扩散速度进行 比较. 如果溶质原子的扩散速度小于钛原子的速度, 则晶界处溶质原子将阻碍晶界的迁移, 此时钛原子 的扩散速度(晶界迁移速度)将降低; 如果溶质原子速 度大于钛原子速度, 那么晶界迁移速度保持不变.

跃迁元胞的长大距离根据方程(8)计算. 长大距 离与元胞尺寸的比值决定元胞是否发生粗化. 若比 值大于 1 , 跃迁元胞的所有状态变量值更新为其长大 方向的邻居元胞的状态变量值, 而且跃迁元胞的长 大距离归值为 0 , 即从新计算下一粗化步的长大距 离. 若比值小于 1 , 则跃迁元胞不发生粗化, 其状 态变量值不发生变化, 元胞长大距离根据方程 (8) 更新.

(3) 微观组织演化. 当晶界元胞发生跃迁和晶 界迁移后, 其状态变量进行更新. 同时, 其邻居元胞 的状态变量值也进行相应更新.

\section{2 模型验证}

本文元胞自动机模型选取 $500 \times 500$ 四边形单元, 采取周期性边界条件, Neumann 邻居类型. 通过元胞 自动机模型模拟 $\mathrm{Ti}-6 \mathrm{Al}-4 \mathrm{~V}$ (TC4), TG6 (Ti-5.8Al
$-4.0 \mathrm{Sn}-4.0 \mathrm{Zr}-0.7 \mathrm{Nb}-1.5 \mathrm{Ta}-0.4 \mathrm{Si}-0.06 \mathrm{C}, \mathrm{wt} \%), \mathrm{Ti} 17$ (Ti-5Al-4Mo-4Cr-2Sn-2Zr, wt\%) 钛合金在单相区晶 粒的静态粗化过程.

\section{1 模拟验证 TC4 钛合金单相区静态粗化}

TC4 钛合金是一种双相钛合金, 其化学成分如 表 1 所示. 表 2 为计算溶质原子扩散速度所需材料参 数. 根据方程(10), TC4 钛合金溶质原子的单相区的 扩散速度如表 3 所示.

根据以上计算结果 (表 3), 溶质原子如铝和杂质 元素原子如碳、氢、氧等的扩散速度远远大于钛原子. 因此, 位于晶界处的溶质原子铝及各类杂质原子不 会阻碍钛原子的扩散, 其拖拽效应可以忽略. 尽管铁 原子的扩散速度小于钛原子的速度, 但是在 $\mathrm{TC} 4$ 钛 合金中铁元素的含量极低, 其拖拽效应也可忽略. 而 beta-钛稳定元素钒, 其原子扩散速度接近于钛原子 速度, 因此其拖拽效应也可近似忽略. 最近, 学者 Gil 等人 ${ }^{[33]}$ 通过试验研究了 $\mathrm{TC} 4$ 钛合金在不同温度下 的粗化特性. 研究发现, TC4 钛合金单相区的晶粒粗 化指数大约在 0.53 0.55 之间, 接近理想粗化指数值 0.5. 因此, TC4 钛合金单相区粗化可以近似认为是一 个理想的粗化过程, 即粗化过程中溶质原子的拖拽 效应可以不计.

本文通过考虑溶质原子的拖拽效应和晶界迁移 各向异性对粗化过程的影响, 建立元胞自动机模型 模拟 TC4 合金单相区的晶粒静态粗化过程. 其中元 胞自动机模型的参数取值包括自扩散激活能、初始晶

表 1 TC4 钛合金化学成分 ${ }^{[33]}$

\begin{tabular}{ccccccccc}
\hline 合金元素 & $\mathrm{Al}$ & $\mathrm{V}$ & $\mathrm{Fe}$ & $\mathrm{C}$ & $\mathrm{O}$ & $\mathrm{N}$ & $\mathrm{H}$ & $\mathrm{Ti}$ \\
\hline $\begin{array}{c}\text { 质量分数 } \\
(\mathrm{wt} \%)\end{array}$ & 6.1 & 4.0 & 0.1 & 0.21 & 0.13 & 0.005 & 0.004 & 其余 \\
\hline
\end{tabular}

表 2 计算 TC4 钛合金溶质原子扩散速度所需材料参数

\begin{tabular}{|c|c|c|c|c|c|c|c|c|}
\hline 溶质原子 & $\mathrm{Ti}$ & $\mathrm{Al}$ & $\mathrm{V}$ & $\mathrm{Fe}$ & $\mathrm{C}$ & $\mathrm{O}$ & $\mathrm{N}$ & $\mathrm{H}$ \\
\hline $\begin{array}{c}\text { 原子半径 } \\
(\mathrm{pm})\end{array}$ & 140 & 125 & 13 & 140 & 70 & 60 & 65 & 21 \\
\hline 原子质量 & 47.87 & 27 & 51 & 56 & 12 & 16 & 14 & 1 \\
\hline
\end{tabular}

表 3 TC4 钛合金溶质原子单相区扩散速度

\begin{tabular}{cccccccc}
\hline 溶质原子 & $\mathrm{Al}$ & $\mathrm{V}$ & $\mathrm{Fe}$ & $\mathrm{C}$ & $\mathrm{O}$ & $\mathrm{N}$ & $\mathrm{H}$ \\
\hline $\begin{array}{c}\text { 扩散速度比 } \\
\left(v_{i} / v_{\mathrm{Ti}}\right)\end{array}$ & 1.98 & 0.97 & 0.855 & 7.98 & 6.98 & 7.36 & 319 \\
\hline
\end{tabular}


粒尺寸、系统温度及粗化时间均采用 Gil 等人 ${ }^{[33]}$ 的实 验数据. 图 2(a) (c) 所示为粗化过程中晶粒尺寸与时 间的关系. 相应的晶粒尺寸与时间的线性关系如图 2(d f) 所示.

模拟结果显示, 粗化过程中晶粒长大速率随着 温度升高而增加. 而在特定温度下, 在初始阶段, 其 长大速率快速增大, 但随着粗化过程的进行, 其长大
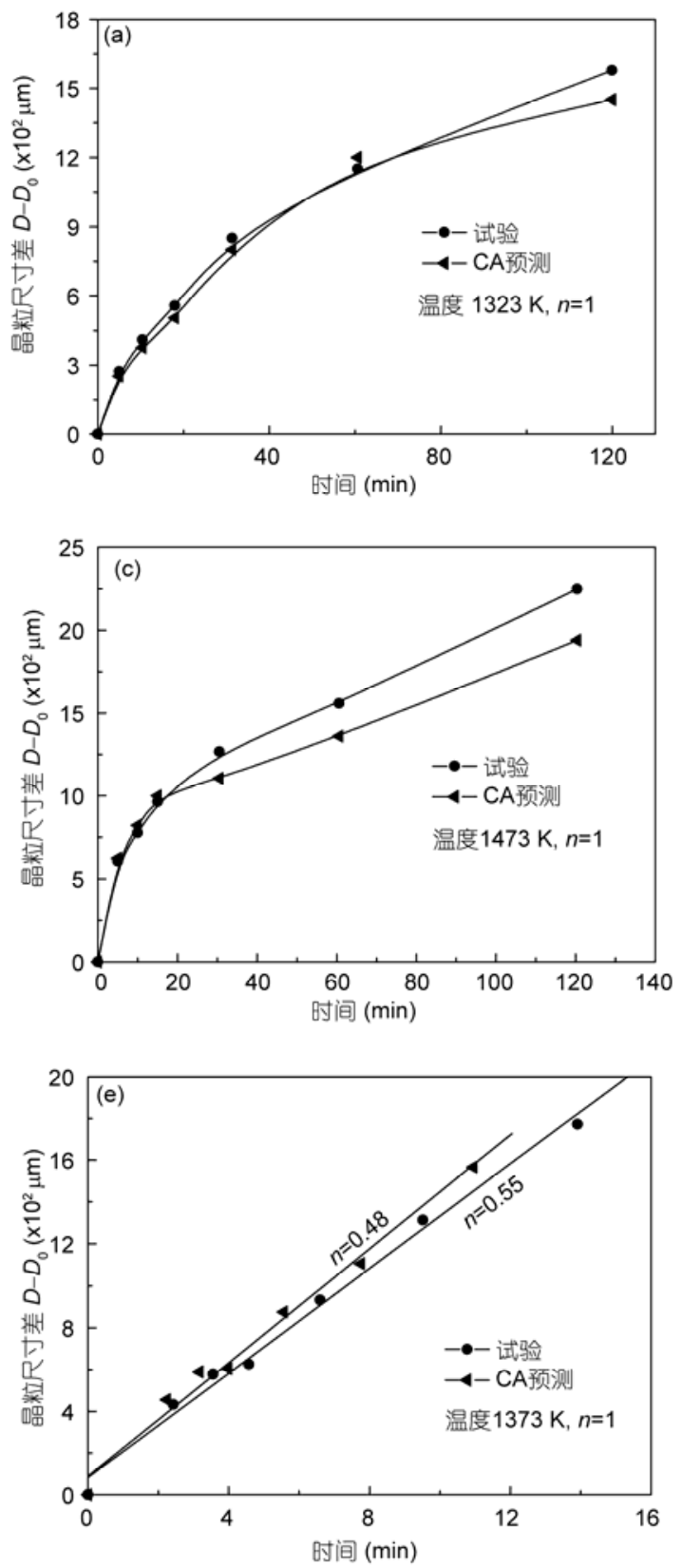

速率逐渐降低. 这一现象主要是由于随着粗化的进 行, 晶粒体积逐渐增加, 从而导致单位体积的晶界面 积降低, 最终导致单位体积的界面能的降低. 因此, 随着时间进行, 晶粒粗化驱动力逐渐降低, 导致随着 粗化时间的增加, 晶粒长大速率逐渐降低. 然而, 由 于本文元胞自动机模型自身的局限性, 与试验结果 相比, 模拟结果仍然存在一定的偏差. 表 4 所示为模
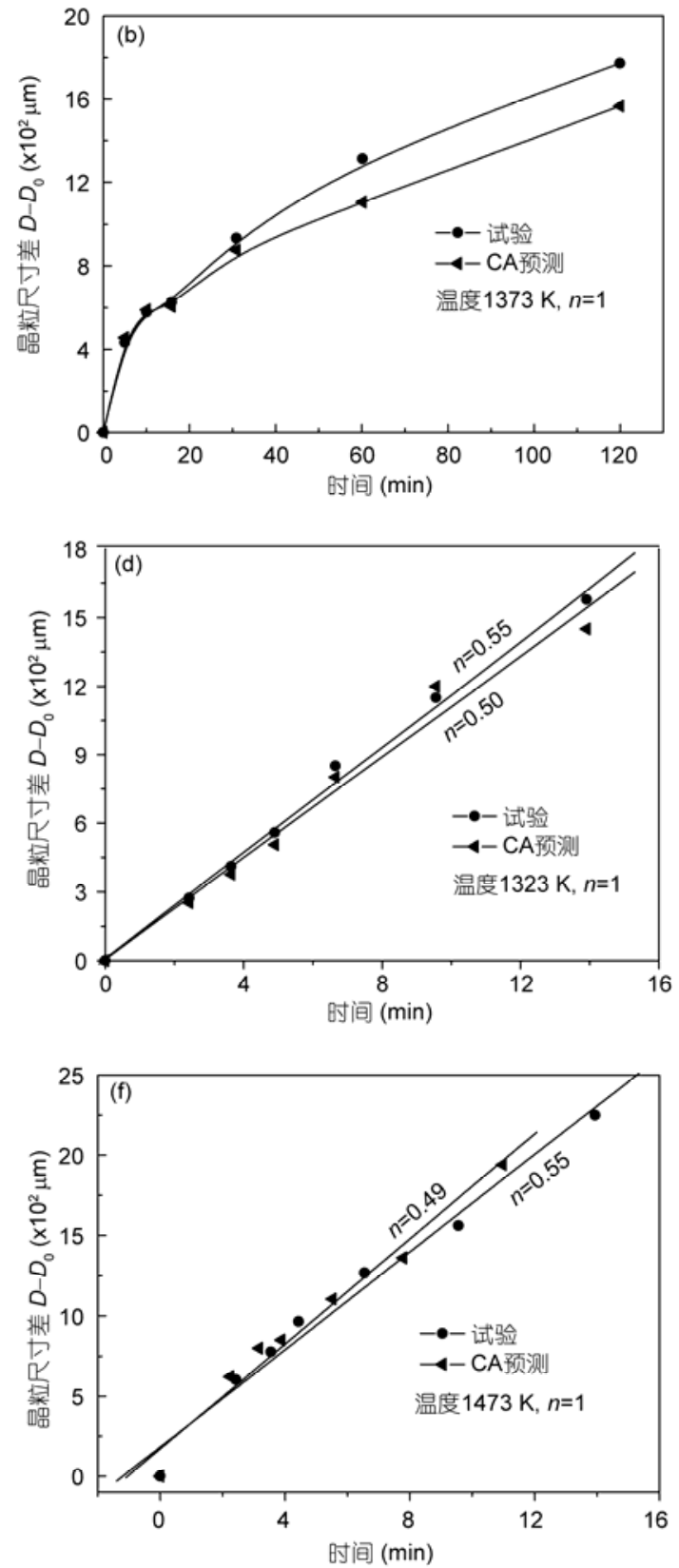

图 2 TC4 钛合金单相区粗化动力学曲线试验与模拟比较

(a) (c)分别表示 TC4 合金在 1323, 1373 和 $1473 \mathrm{~K}$ 条件下晶粒粗化晶粒尺寸与时间的关系, $n=1$; (d) (f)分别表示 TC4 合金在 1323,1373 和 1473 $\mathrm{K}$ 条件下粗化晶粒尺寸与时间的线性关系 
表 4 在不同温度下, TC4 钛合金单相区粗化指数的预测 值与试验值比较

\begin{tabular}{cccc}
\hline 温度 $(\mathrm{K})$ & 1323 & 1373 & 1473 \\
\hline $\begin{array}{c}\text { 粗化指数 } \\
\text { (试验值/预测值) }\end{array}$ & $0.55 / 0.50$ & $0.55 / 0.48$ & $0.55 / 0.49$ \\
相对误差 $(\%)$ & 9 & 13 & 11 \\
\hline
\end{tabular}

拟所得粗化指数与试验值的比较.

\section{2 模拟验证 TG6 和 Ti17 钛合金单相区晶粒 静态粗化}

TG6 是一类新的近 alpha 高温钛合金, 主要应用 于航空发动机材料 ${ }^{[3]}$. Ti17 是一种近 beta 钛合金. Wang 等人 ${ }^{[3]}$ 通过试验研究了这两种钛合金在等温条 件下单相区粗化现象. 研究发现, 对于 TG6 钛合金, 其粗化指数取值范围为 $0.35 \sim 0.38$, 而对于 Ti17 钛合 金, 其粗化指数的取值范围为 $0.27 \sim 0.30$. 通过试验, 同样计算了这两种合金的自扩散激活能, 取值范围 分别为 $120.4 \sim 212.5 \mathrm{~kJ} / \mathrm{mol}$ 和 $290.5 \sim 378.5 \mathrm{~kJ} / \mathrm{mol}$. 通 过试验数据计算得到的粗化指数和自扩散激活能与 理想粗化过程的粗化指数 $(0.5)$ 和激活能 $(97 \mathrm{~kJ} / \mathrm{mol})$. 溶质原子对迁移晶界的阻碍可能导致粗化指数的降 低及激活能的升高.

考虑溶质原子拖拽效应及晶界迁移各向异性的 元胞自动机模型用以模拟钛合金单相区的粗化. TG6
和 Ti17 钛合金化学成分及计算溶质扩散速度的材料 常数如表 5, 6 所示. 根据方程(10), TG6 和 Ti17 钛 合金溶质原子与钛原子的扩散速度比值如表 7 所示. 图 3(a) (c) 与图 4(a) (c) 所示为 TG6 和 Ti17 合金单相 区粗化过程中晶粒尺寸与粗化时间的关系. 如图 3(d f) 和图 4(d f) 所示, 模拟所得粗化指数与试验值 较为吻合. 对于 TG6 合金, 其模拟与试验计算所得 粗化指数的最大相对误差为 $13.2 \%$, 合金 Ti17 的最 大相对误差为 $20.6 \%$. 表 8 所示为元胞机预测粗化指 数值与试验值之间的相对误差

\section{3 结果与讨论}

\section{1 温度与时间对微观组织的影响}

图 5 所示为 TA15 (Ti-6Al-2Zr-1Mo-1V)钛合金在 $1313 \mathrm{~K}$ 保温时间从 10 30 min 的过程中微观组织的 演化. 其中, 图 5(a c) 为元胞机模拟晶粒粗化过程微 观组织演化, 图 5(d f) 为试验观察. 从图中晶粒尺寸 的变化可以看出, 粗化时间对微观组织演化的影响 很大. 元胞机模拟平均晶粒尺寸的变化范围大约从 $10 \mathrm{~min}$ 的 $300 \mu \mathrm{m}$ (直径)增加到保温 $30 \mathrm{~min}$ 的 $600 \mu \mathrm{m}$. 通过试验观察, TA15 合金在同样条件下粗化过程微 观组织演化如图 5(d) (f) 所示, 其平均晶粒尺寸从 410 增加到 $630 \mu \mathrm{m}$. 试验所得各自保温时间下的晶粒

表 5 钛合金 TG6, Ti17 的化学成分 $(w t \%)$

\begin{tabular}{ccccccccccccccc}
\hline 合金元素 & $\mathrm{Al}$ & $\mathrm{Sn}$ & $\mathrm{Zr}$ & $\mathrm{Mo}$ & $\mathrm{Cr}$ & $\mathrm{Nb}$ & $\mathrm{Ta}$ & $\mathrm{Si}$ & $\mathrm{C}$ & $\mathrm{Fe}$ & $\mathrm{N}$ & $\mathrm{O}$ & $\mathrm{Ti}$ \\
\hline TG6 & 5.74 & 3.94 & 3.78 & - & - & 0.64 & 1.61 & 0.45 & 0.054 & 0.03 & 0.008 & 0.064 & 其余 \\
Ti17 & 5.05 & 2.13 & 2.07 & 4.12 & 4.13 & - & - & - & 0.01 & 0.04 & 0.002 & 0.08 & 其余 \\
\hline
\end{tabular}

表 6 钛合金 TG6, Ti17 的溶质原子半径及原子质量

\begin{tabular}{|c|c|c|c|c|c|c|c|c|c|c|c|c|c|}
\hline 合金元素 & $\mathrm{Al}$ & $\mathrm{Sn}$ & $\mathrm{Zr}$ & Mo & $\mathrm{Cr}$ & $\mathrm{Nb}$ & $\mathrm{Ta}$ & $\mathrm{Si}$ & $\mathrm{C}$ & $\mathrm{Fe}$ & $\mathrm{N}$ & $\mathrm{O}$ & $\mathrm{Ti}$ \\
\hline 半径 (pm) & 125 & 145 & 155 & 145 & 140 & 145 & 145 & 110 & 70 & 140 & 65 & 60 & 140 \\
\hline 质量 & 27 & 119 & 91 & 96 & 52 & 93 & 181 & 28 & 12 & 56 & 14 & 16 & 47.87 \\
\hline
\end{tabular}

表 7 钛合金 TG6, Ti17 的溶质原子与钛原子扩散速度之比

\begin{tabular}{|c|c|c|c|c|c|c|c|c|c|c|c|c|}
\hline 合金元素 & $\mathrm{Al}$ & $\mathrm{Sn}$ & $\mathrm{Zr}$ & Mo & $\mathrm{Cr}$ & $\mathrm{Nb}$ & $\mathrm{Ta}$ & $\mathrm{Si}$ & $\mathrm{C}$ & $\mathrm{Fe}$ & $\mathrm{N}$ & $\mathrm{O}$ \\
\hline 比值 $\left(v_{i} / v_{\mathrm{Ti}}\right)$ & 1.98 & 0.388 & 0.475 & 0.48 & 0.92 & 0.497 & 0.255 & 2.18 & 7.97 & 0.855 & 7.36 & 6.98 \\
\hline
\end{tabular}

表 8 在不同温度下, 钛合金 TG6 与 Ti17 单相区粗化指数预测值与试验值对比

\begin{tabular}{|c|c|c|c|c|c|c|}
\hline 合金 & 1323 & 1423 & 1473 & 1178 & 1188 & 1198 \\
\hline 温度 $(\mathrm{K})$ & & TG6 & & & Ti17 & \\
\hline 粗化指数(试验/模拟) & $0.35 / 0.35$ & $0.37 / 0.34$ & $0.38 / 0.33$ & $0.27 / 0.30$ & $0.29 / 0.35$ & $0.30 / 0.35$ \\
\hline 相对误差(\%) & 0 & 8 & 13.2 & 11.1 & 20.6 & 16.7 \\
\hline
\end{tabular}



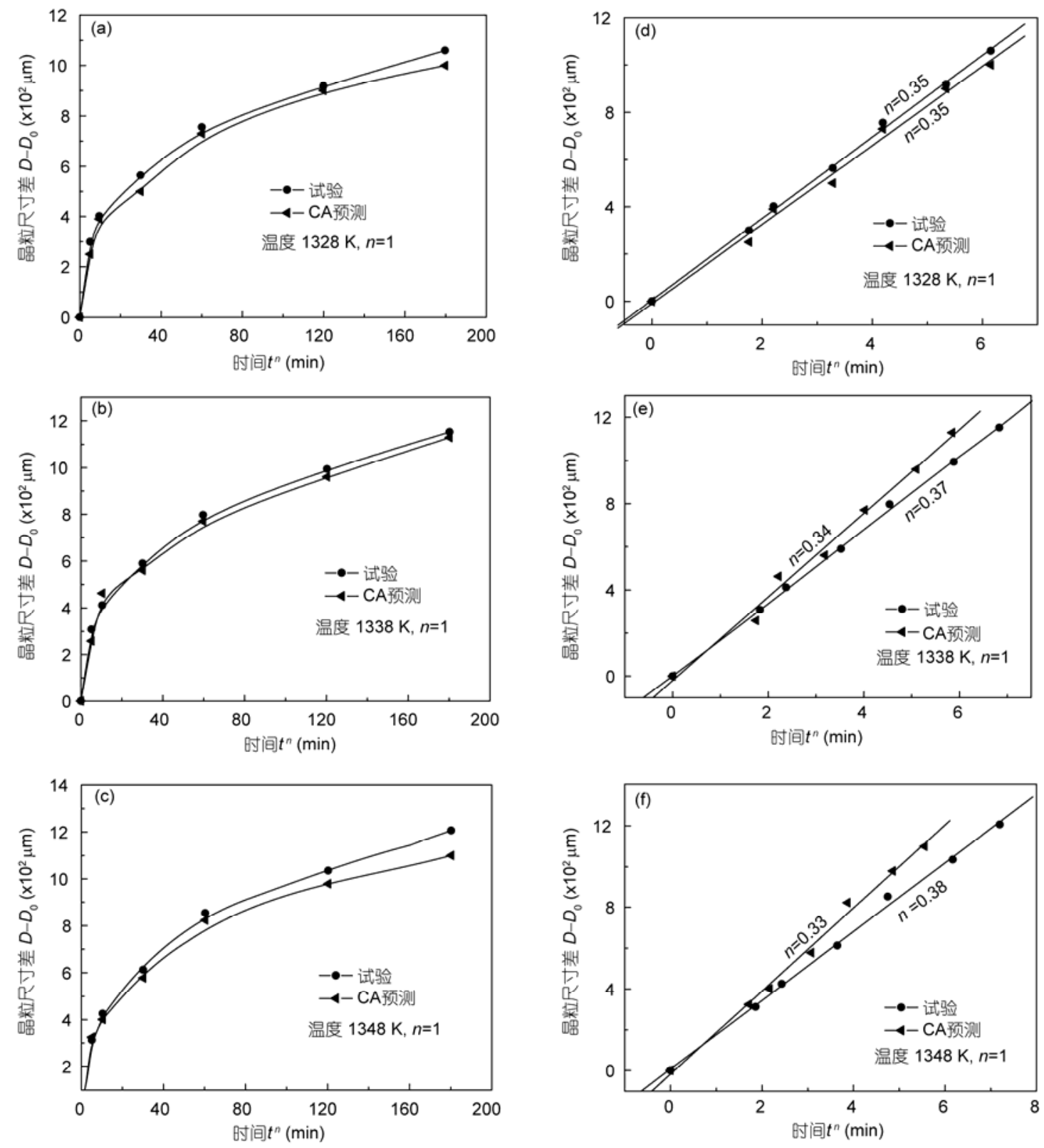

图 3 合金 TG6 单相区粗化动力学模拟与试验对比

(a) (c)分别表示 TG6 合金在 1328, 1338 和 $1348 \mathrm{~K}$ 条件下晶粒粗化晶粒尺寸与时间的关系, $n=1$; (d) (f)分别表示 TG6 合金在 1328, 1338 和 $1348 \mathrm{~K}$ 条件下粗化晶粒尺寸与时间的线性关系

尺寸与模拟存在偏差. 在初始阶段, 即保温 $10 \mathrm{~min}$, 试验所得晶粒尺寸大约为 $410 \mu \mathrm{m}$, 其值远远大于模 拟所得晶粒尺寸 $300 \mu \mathrm{m}$. 较大误差的存在可能是由 于初始阶段元胞机模拟微观组织演化处于不稳定状 态, 晶粒数目变化较大, 从而导致模拟与试验之间误 差较大. 随着粗化时间的增加, 微观组织逐渐区域稳 定, 即晶粒数目变化相对较小, 所以模拟所得晶粒尺 寸与试验观察值之间的误差较初始阶段较小. 在粗
化过程中, 拓扑特征的演化几乎不受保温时间和温 度的影响. Raghavan 等人 ${ }^{[22,23]}$ 及 Chen 等人 ${ }^{[26]}$ 通过元 胞自动机模型模拟晶粒的粗化过程, 研究发现边数 大于 6 的晶粒的长大以吞噬边数小于 6 的晶粒为代价. 而且随着粗化的进行, 大多数晶粒的边数等于 6 , 组 织结构逐渐趋于稳定. 这与 von Neumann-Mullins 理 论相符, 即晶粒的长大速率与其边数相关, 而且在整 个粗化过程中, 晶粒边数的分布与时间、温度无关. 

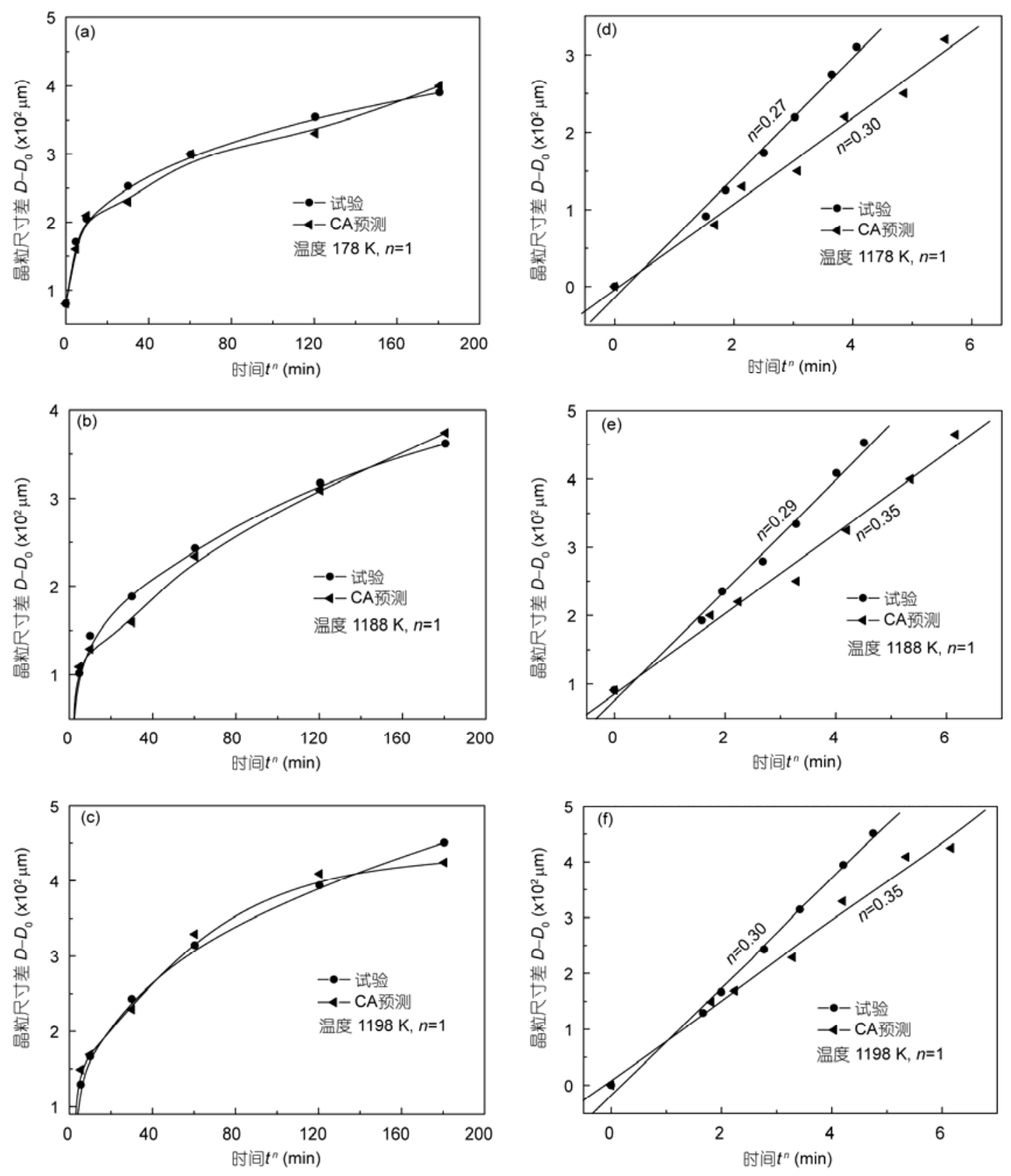

图 4 合金 Ti17 单相区粗化动力学模拟与试验对比

(a) (c)分别表示 Ti17 合金在 1178, 1188 和 $1198 \mathrm{~K}$ 条件下晶粒粗化晶粒尺寸与时间的关系, $n=1$; (d) (f)分别表示 Ti17 合金在 1178,1188 和 $1198 \mathrm{~K}$ 条件下粗化晶粒尺寸与时间的线性关系

由于统计误差或者元胞自动机算法自身的缺陷, 元 胞机模拟预测晶粒边数的分布与试验统计之间存在 偏差.

\section{2 合金化学成分对粗化动力学的影响}

合金化学成分严重影响材料的粗化动力学过程.
在单相区, TC4 钛合金的粗化指数的分布范围为 $0.5 \sim 0.55^{[33]}$, 接近粗化指数的理论值 0.5 . 对于合金 TG6 和 Ti17, 单相区粗化指数值远远小于 TC4 合金 的粗化指数值, 这主要是由于溶质原子的拖拽效应. 对于 TC4 钛合金, 溶质原子主要包括铝和钒. 铝原子 与钛原子扩散速度之比为 0.97 . 而钒元素, 其原子 


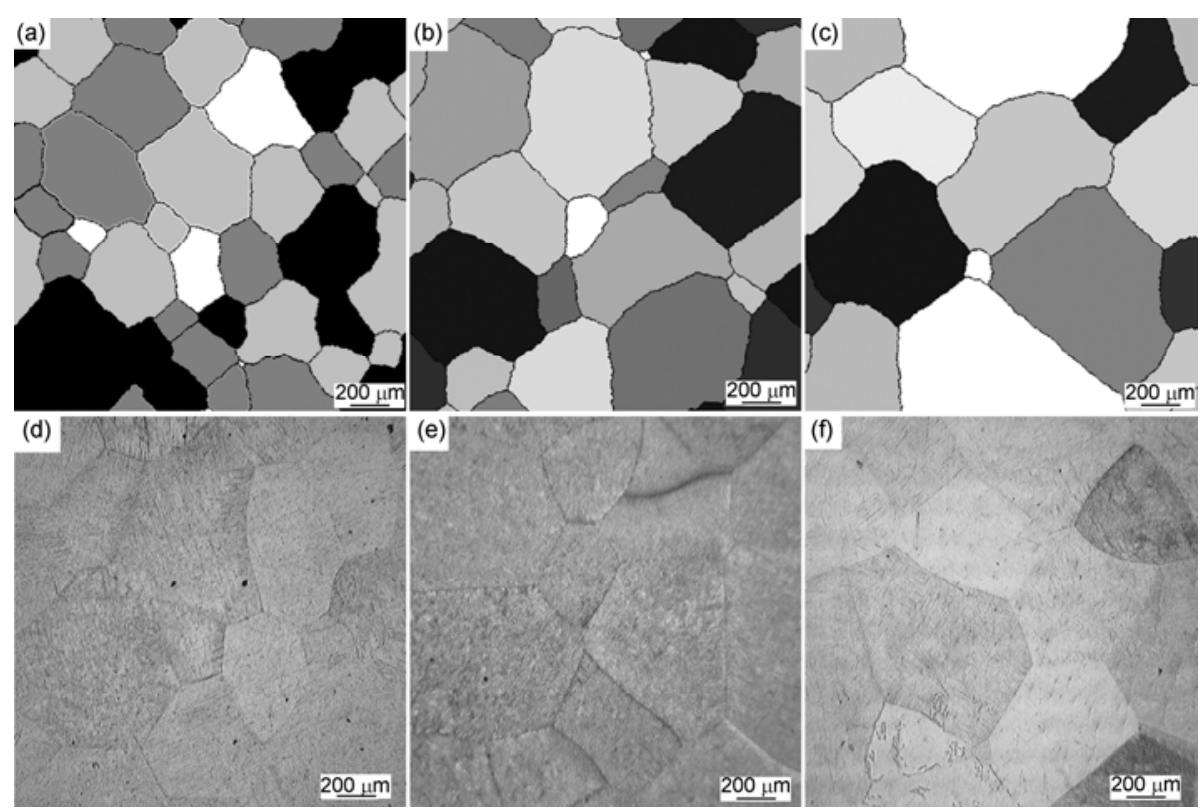

图 5 TA15 钛合金在温度为 $1313 \mathrm{~K}$ 不同保温时间下粗化过程微观组织演化元胞自动机模拟(a) (c) 与试验 (d) (f) 对比

(a), (d) $10 \mathrm{~min}$; (b), (e) $30 \mathrm{~min}$; (c), (f) $60 \mathrm{~min}$

的扩散速度大于钛原子速度(比值为 1.98). 对于 TC4 钛合金, 铝与钒溶质原子的扩散速度不小于钛原子 的速度. 因此, 在粗化过程中, 这两类溶质原子对晶 界的拖拽效应可以忽略不计. 尽管 TC4 合金中杂质 元素铁的原子扩散速度小于钛原子, 但是其含量很 低, 所以仍可忽略其拖拽效应. 因此, TC4 单相区晶 粒粗化指数接近理论值 0.5 . 然而对于 TG6 和 Ti17 钛合金, 溶质原子的拖拽效应明显影响晶粒的粗化 指数. 通过表 6 可知, 溶质原子如锡、锆、钿、铬、 镍, 其原子半径和原子质量均远远大于钛原子的. 因 此, 根据方程(10) (12), 这些溶质原子与钛原子扩散 速度之比均小于 1 , 如表 7 所示. 这说明在粗化过程 中, 这些溶质原子将阻碍晶界的迁移. 因此, 通过模 拟, TG6 和 Ti17 合金单相区粗化指数的分布范围在 0.27 0.38 之间, 小于理论值 0.5 , 与试验结果相符.

\section{3 模型的局限性}

本文通过考虑溶质原子拖拽效应及晶界迁移率 各向异性对粗化的影响, 建立了元胞自动机模型, 用 以模拟钛合金在单相区的静态粗化过程. 本文模型 主要用以预测温度和保温时间对粗化过程的影响. 在这一过程中, 模拟研究了粗化动力学、晶粒尺寸随 时间与温度的变化关系, 预测结果与相应的试验结 果进行了比较. 影响粗化的其他因素有应变与应变
率. 由于塑性变形引起的应变提高了变形晶粒的塑 性能, 从而改变了晶粒粗化的动力学过程或改变了 粗化指数. 应变率的变化会影响动态粗化过程, Semiatin 等人 ${ }^{[8]}$ 已经通过大量的试验研究了 TC4 钛合 金的动态粗化过程, 并得到了一些重要结论. 但是, 本文仅仅是通过元胞自动机法模拟双相钛合金单相 区的静态粗化过程. 因此, 没有考虑应变及应变率对 粗化的影响. 关于通过元胞自动机算法模拟动态粗 化并考虑应变及应变率的影响, 将是本课题的下一 步研究内容.

为了验证模型的准确性, 其中模型预测的粗化 指数、晶粒尺寸与时间动力学关系及组织演化与相应 的试验值进行了对比, 吻合较好, 但是仍然存在一定 的偏差. 如预测晶粒粗化指数与试验结果之间的平 均偏差为 $11 \%$ 13\%, 最大偏差为 $20.7 \%$ (Ti17 钛合金, $1188 \mathrm{~K})$. 下列因素导致了模拟值与试验值之间存在 偏差. 首先是由于元胞自动机算法本身缺陷带来的 不可避免的误差, 元胞机算法是将连续问题离散化, 这势必带来误差. 其次是由于方程(10)本身的缺陷, 如计算精度低, 或者影响溶质扩散速度的因素没有 完全考虑, 这也是模拟中最大的误差源. 另外, 影响 粗化过程的其他因素如第二相粒子, 析出物及微观 结构缺陷等都没有考虑. 上述因素导致本文的预测 结果与试验结果之间存在一定的偏差. 


\section{4 结论}

本文通过考虑溶质原子的拖拽和晶界迁移率各 向异性对粗化的影响，建立了元胞自动机模型，模拟 晶粒的静态粗化. 为了定量描述溶质原子的拖拽效 应对粗化的影响, 其扩散速度等效转化为钛原子的 速度. 为此, 本文提出了一个描述它们之间转化关系
的数学表达式. 在式中, 考虑溶质原子的半径、原子 质量及晶格类型对扩散速度的影响. 另外通过引人 参数 $c_{0}$ 考虑晶界迁移率各向异性对粗化的影响. 文 中将元胞自动机模型用以模拟 TC4，TG6，TA15 及 Ti17 钛合金单相区晶粒粗化过程，并将模拟预测值 包括粗化指数、晶粒尺寸与时间关系及微观组织演化 与试验进行了对比, 结果表明较为吻合.

\section{参考文献}

1 Eylon D, Hall S A, Pierce C M, et al. Microstructure and mechanical properties relationships in the Ti-11 alloy at room and elevated temperatures. Metall Mater Trans A, 1976, 7: 1826-1871

2 Zhang M, Zhang J, McDowell D L. Microstructure-based crystal plasticity modeling of cyclic deformation of Ti-6Al-4V. Int J Plast, 2007, 23: $1328-1348$

3 Wang T, Guo H Z, Tan L J, et al. Beta grain growth behaviour of TG6 and Ti17 titanium alloys. Mater Sci Eng A, 2011, 528: 6375-6380

4 Ivasishin O M, Shevchenko S L, Semiatin S L. Effect of crystallographic texture on the isothermal beta grain-growth kinetics of Ti-6Al-4V. Mater Sci Eng A, 2002, 332: 343-350

5 Ivasishin O M, Semiatin S L, Markovsky P E, et al. Grain growth and texture evolution in Ti-/6Al-4V during beta annealing under continuous heating conditions. Mater Sci Eng A, 2002, 337: 88-96

6 Semiatin S L, Fagin P N, Glavicic M G, et al. Influence on texture on beta grain growth during continuous annealing of Ti-6Al-4V. Mater Sci Eng A, 2001, 299: 225-234

7 Semiatin S L, Kirby B C, Salishchev G A. Coarsening behavior of an alpha-beta titanium alloy. Metall Mater Trans A, 2004, 35: 2809-2819

8 Semiatin S L, Corbett M W, Fagin P N, et al. Dynamic-coarsening behavior of an alpha/beta titanium alloy. Metall Mater Trans A, 2006, 37: $1125-1136$

9 Bradley J R, Rigsbee J M, Aaronson H I. Growth kinetics of grain boundary ferrite allotriomorphs in Fe-C alloys. Metall Mater Trans A, 1977, 6: 323-333

10 Cotrina E, Iza-Mendia A, López B. Study of the ferrite grain coarsening behind the transformation front by electron backscattered diffraction techniques. Metall Mater Trans A, 2004, 35: 93-102

11 Tong M M, Li D Z, Li Y Y. Modeling the austenite-ferrite diffusive transformation during continuous cooling on a mesoscale using Monte Carlo method. Acta Mater, 2004, 52: 1155-1162

12 Tong $\mathrm{M} \mathrm{M}$, Ni J, Zhang Y, et al. A new Monte Carlo simulation of three-dimensional microstructures and their evolution in polycrystalline. Scr Mater, 2004, 50: 909-913

13 Song X Y, Liu G Q, Gu N J. A new Monte Carlo simulation of three-dimensional microstructures and their evolution in polycrystalline. Chin Sci Bull, 1999, 44: 1432-1436

14 Luo B C, Wang H P, Wei B B. Phase field simulation of monotectic transformation for liquid Ni-Cu-Pb alloys. Chin Sci Bull, 2009, 54: $183-188$

15 Zaeem M A, Kadiri H E, Wang P T, et al. Investigating the effects of grain boundary energy anisotropy and second-phase particles on grain growth using a phase-field model. Comput Mater Sci, 2011, 50: 2488-2492

16 Zheng C W, Xiao N M, Li D Z, et al. Microstructure prediction of the austenite recrystallization during multi-pass steel strip hot rolling: A cellular automaton modeling. Comput Mater Sci, 2008, 44: 507-514

17 Zheng C W, Xiao N M, Li D Z. Mesoscopic modeling of austenite static recrystallization in a low carbon steel using a coupled simulation method. Comput Mater Sci, 2009, 45: 568-575

18 Ding R, Guo Z X. Microstructural modeling of dynamic recrystallization using an extended cellular automaton approach. Comput Mater Sci, 2002, 23: 209-218

19 Yang H, Wu C, Li H W, et al. Review on cellular automata simulations of microstructure evolution during metal forming process: Grain coarsening, recrystallization and phase transformation. Sci China Tech Sci, 2011, 54: 2107-2118

20 Ding H L, He Y Z, Liu L F. Cellular automata simulation of grain growth in three dimensions based on the lowest-energy principle. J Cryst Growth, 2006, 293: 489-497 
21 Geiger J, Roósz A, Barkóczyb P. Simulation of grain coarsening in two dimensions by cellular automaton. Acta Mater, 2001, 49: 623-629

22 Raghavan S, Sahay S S. Modeling the grain growth kinetics by cellular automaton. Mater Sci Eng A, 2007, 445-446: 203-209

23 Raghavan S, Sahay S S. Modeling the topological features during grain growth by cellular automaton. Comput Mater Sci, 2009, 46: 92-99

24 Kugler G, Turk R. Study of the influence of initial microstructure topology on the kinetics of static recrystallization using a cellular automata model. Comput Mater Sci, 2006, 37: 284-291

25 Mao W M, An Z G, Li S X. Influence of MnS particles on the behaviors of grain boundary migration in Fe-3\%Si alloys. Chin Sci Bull, 2009, 54: 4537-4540

26 Chen F, Cui Z S, Liu J. Mesoscale simulation of the high-temperature austenitizing and dynamic recrystallization by coupling a cellular automaton with a topology deformation technique. Mater Sci Eng A, 2010, 527: 5539-5549

27 He Y Z, Ding H L, Liu L F, et al. Computer simulation of 2D grain growth using a cellular automata model based on the lowest energy principle. Mater Sci Eng A, 2006, 429: 236-246

28 Yazdipour N, Davies C H J, Hodgson P D. Microstructural modeling of dynamic recrystallization using irregular cellular automata. Comput Mater Sci, 2008, 44: 566-576

29 Chen F, Cui Z S, Liu J. Modeling and simulation on dynamic recrystallization of 30Cr2Ni4MoV rotor steel using the cellular automaton method. Model Simul Mater Sci Eng, 2009, 17: 1-19

30 Kugler G, Turk R. Modeling the dynamic recrystallization under multi-stage hot deformation. Acta Mater, 2004, 52: 4659-4668

31 Fjeldberg E, Marthinsen K. A 3D Monte Carlo study of the effect of grain boundary anisotropy and particles on the size distribution of grains after recrystallisation and grain growth. Comput Mater Sci, 2010, 48: 267-281

32 Chun Y B, Semiatin S L, Hwang S K. Monte Carlo modeling of microstructure evolution during the static recrystallization of cold-rolled, commercial-purity titanium. Acta Mater, 2006, 54: 3673-3689

33 Gil F J, Planell J A. Behaviour of normal grain growth kinetics in single phase titanium and titanium alloys. Mater Sci Eng A, 2000, 283: $17-24$ 